\title{
NEW IP Framework and Protocol for Future Applications
}

\author{
Zhe Chen, Chuang Wang, Guanwen Li, Zhe Lou, Sheng Jiang \\ Huawei Technologies Co., Ltd. \\ \{chenzhe17, wangchuang, liguanwen, zhe.lou, jiangsheng\}@ huawei.com
}

\author{
Alex Galis \\ University College London \\ a.galis@ucl.ac.uk
}

\begin{abstract}
It is observed that the Internet has been slowly "fragmented" into Many Networks (ManyNets) due to both technical and commercial evolutions. The interconnection among those networks is a challenge due to their incompatible addressing mechanisms. Furthermore, emerging applications like Augmented Reality and Virtual Reality (AR/VR), holographic communications, etc. rise new requirements to the network and involve innumerable physical and virtual objects, which require more efficient and customized network services. Current Internet Protocol (IP) that aims to connect regional academic and military networks was invented half a century ago. The original IP design philosophy like fixed address length, binding machines to specific locations, and others are not sufficient anymore to tackle those challenges.

In this paper, we propose a novel framework for future Internet protocol, called NEW IP, to tackle aforementioned challenges and fulfill the requirements of future applications. NEW IP inherently enables variable-length and multi-semantic network addresses, and user-definable networking. Besides the NEW IP framework, a few use cases will be illustrated to highlight the benefits it brings.

Index Terms-Future Internet, ManyNets, NEW IP Framework, Flexible Addressing, Service-Aware Routing, UserDefinable Networking
\end{abstract}

\section{INTRODUCTION}

Novel technologies and applications are the driving force to the evolution of the Internet. Among all the others, the ManyNets [1], the holographic communication [2] and the huge amount of ever-increasing physical and virtual objects on the Internet, may give a big impact on the existing IP protocol and framework. First of all, the emerging and growth of ManyNets including the Internet of Things (IoT) network, the cellular network, the industrial network, the satellite network, etc., makes the Internet "fragmented". The interconnectivity among ManyNets in order to make information and services from one network available (if permitted) to another network becomes a challenging task. Although it was designed for the interconnection of several regional academic and military networks in the 1970s, the current TCP/IP protocols and framework contain limitations for the ManyNets interconnectivity. For instance, the fixed IP address length, either 32 bits for IPv4 or 128 bits for IPv6, might not be suitable for all networks. The IoT network typically asks for shorter addresses to reduce the power, computation and memory consumption. Some industrial networks even "remove" the IP header in

978-1-7281-4973-8/20/\$31.00 @ 2020 IEEE order to increase the communication efficiency. The spaceground communication can hardly use the existing fixed IP addressing strategy of binding of the subnet and route port due to the high dynamic movement of the satellite nodes. Although the IPv6 over Low Power Wireless Personal Area Networks (6LoWPAN) [3] tackled some of the issues, the complexity brought by its header compression mechanism is unacceptable. Thus, a more dynamic IP addressing solution is required.

Holographic communication that meets human being's desires on tangible, immersive and interactive communication experience, demands higher bandwidth, lower latency, and flexible network processing. Technologies like priority queue management and the per-flow scheduling are neither efficient nor scalable, thus not sufficient to meet those requirements. A more fine-grained network service capable of sensing the needs of applications, is required to process each individual packet with different methods that should be defined by users or applications in order to achieve the best Quality of Experience (QoE) [4].

The Internet is experiencing ever-increasing physical and virtual objects including personal computers, mobile handsets, sensors, contents, services, capabilities, and other virtual entities. The current IP address was originally designed to identify physical objects being bounded to specific locations. It doesn't contain the identification of virtual objects (contents, services, etc.), thus is not aware of the content or services it carries, which hinders it from providing the most proper forwarding solution. Various mapping mechanisms were introduced, which not only brings extra complexity to the system, but also raise the potential privacy and resilience issues. Information-Centric Networking (ICN) is a solution proposed by [5] for better content delivery. However, it doesn't take service or other virtual entities into account. Moreover, hierarchical names weaken the routing aggregate-ability for mobile contents. Therefore, a better solution that is capable of taking care of the delivery and communication of all kinds of virtual objects is required.

In this paper, we propose a novel framework for future Internet protocol, called NEW IP, which contains the following three native integrated key features:

- Variable IP address in length to seamlessly support crossnetwork communication;

- Semantic definition of the IP address to identify both physical and virtual objects; 
- User-Definable IP header allowing end-users to specify customized functions to be performed on data packets.

The remainder of this article is arranged as follows: Section 2 reviews the related work. In section 3, the framework of NEW IP is introduced. Section 4 describes a set of use cases to explain the advantages of the proposed architecture. The conclusion and future work are given in section 5.

\section{RELATED WORK}

6LoWPAN is one of the potential technologies to support a large number of IoT devices with low power consumption. It designs an adaptation layer under the network layer to compress the IPv6 header, which reduces the overhead by removing all unused fields and pre-configuring some generic fields with default values. Although the header compression reduces power consumption in communication, it dramatically increases the demands on storage and computation resources due to the complexity in implementation [6].

Named Data Networking (NDN) [7] is a well-known architecture of the ICN, which engages two types of packets: interest packet and data packet. NDN adopts this requestreply communication model and directly uses application data names at the network layer to make network services best match application communication patterns. In NDN network layer, there is no address but the application-oriented namespaces. It adopts hierarchical content names that are suitable for aggregation. However, the hierarchical names would increase the complexity of performing lookups in routers. Moreover, although hierarchical names could reduce the FIB size by aggregation, the aggregate-ability of hierarchical names will be diminished when the content is moving [8].

MobilityFirst [9] is another ICN network architecture, which focuses on mobility support. Since the IP address is multiplexed at both the transport layer and network layer, serving as an identifier and a locator, the conventional IP architecture cannot maintain an end-to-end connection for mobile users. Thus, MobilityFirst defines a flat and globally unique identifier GUID to each network object, which is independent of its network address. By employing a fast global name resolution service (GNRS) to dynamically bind the destination GUID to a current set of network addresses. Though the flat addressing schema of MobilityFirst seamlessly supports devices movement and decreases the complexity of name lookups, the fixedlength address is unfriendly to low-power devices, especially for IoT scenarios.

Variable Length Addressing schemes were analyzed in [14], [15] and [16]. Service-aware routing and a level of flexibility in User-Definable Networking was analyzed in [17], [18].

\section{THE NEW IP FRAMEWORK}

This section presents the design principle of the proposed NEW IP framework. In particular, it illustrates a novel network-layer packet header enabling the key features of the NEW IP being variable address length, multi-semantic addressing, and user-definable networking.
The basic idea is of injecting meta-information into packets/headers in order to provide programmability for network nodes on how to process those packets as enablers of the three native integrated key features. In particular network aware service-related information in the header, which are used to calculate routing tables would replace the traditional best effort service provision approach with service/application considered with network conditions together to achieve a better QoE for users and/or guarantees for KPIs or QoS as required by future precision applications.

Fig. 1 shows the abstract structure of the NEW IP packet header, which is indicated by specific layer- 2 header's next protocol fields (e.g., ETH_TYPE field in the Ethernet header). The NEW IP header starts with a Fields Indicator (FI) that indicates which field(s) are encapsulated in the header. The FI field could be implemented by using a bitmap, where each bit reflects whether a corresponding field appears in the header. It is worth noting that all other fields in the NEW IP header are optional except the FI.

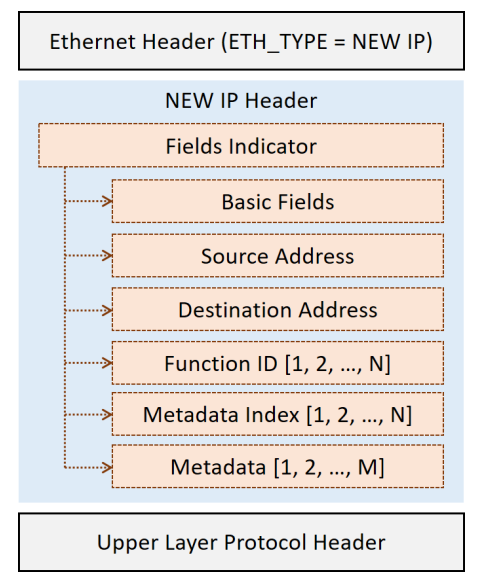

Fig. 1. NEW IP Header Structure.

The FI is followed optionally by a number of basic fields such as Traffic Class, Flow Label, Payload Length, Next Protocol and Time to Live.

Different from the IPv4 or IPv6 headers, the source and destination addresses in the NEW IP packet header are optional and are variable in length. Moreover, the addresses could be defined with multiple semantics. A possible way to achieve such flexibility is to borrow the methodology of Concise Binary Object Representation (CBOR) [10]. The first octet of the address indicates the structures and semantics of the address. Particularly, we utilize a portion of the first octet values, i.e., zero to $\mathrm{x}(\mathrm{x}<255)$, to directly represent 8-bit short addresses. For example, if the first octet of the address is $0 \mathrm{x} 02$, the length of the address is only 8 bits and the address value is 2. To represent long addresses, we utilize specific values (except for zero to $\mathrm{x}$ ) of the first octet to indicate different address lengths. For instance, if the first octet of the address is $0 x F 4$, a 128-bit length address is followed. In addition, to achieve multi-semantic addressing, a specific value of the first octet is used to represent that there is an 8-bit address 
type followed, indicating that a specific type of address (e.g., service, content, user ID) is carried. Moreover, we also use a specific first octet value to indicate that 2 segments of variable length, multi-semantic addresses are followed, thus making the addressing more flexible.

Moreover, NEW IP header might optionally (based on user's requirements) carry Function ID (FID), Metadata Index (MDI), and Metadata (MD) fields to enable user-definable networking. In particular, FIDs represent the functions that an end-user expects the network to perform on the packet. For instance, the function could be "forwarding the packet before a deadline", or "collecting the maximum queue depth along the forwarding path". MDs are corresponding metadata associated with the FIDs listed in the header. For example, the MD could be "the deadline of the packet", or "the maximum queue depth value along the forwarding path". Additionally, MDI is the "bridge" between FID and MD and helps routers to instantly locate the corresponding MD for a particular FID, which is essentially byte-offset (or location) of the MD.

\section{NEW IP USE CASES}

The following use cases highlight the need for Variable IP address, Semantic definition of the IP address, User-definable IP header, flexibility in programming of IP's header and their integration as native features.

\section{A. Flexible Addressing for Smart Home and Edge IoT Systems}

In a home automation system, people can control house lighting, climate, appliances, entertainment systems, and security systems based on collected sensor information. In this subsection, we illustrate how NEW IP achieves flexible addressing in this scenario.

As shown in the Fig. 2, when the motion sensor detects an abnormal activity in the room, it will notify the security camera of the corresponding room to collect image information through the smart home network. Then the security camera sends the collected image information to the host's smartphone via the Internet, assuming the host is not at home. The host will judge whether there is a thief invasion based on the real-time image information. If it is just a false alarm (such as the pet activity), the host may send a message to the security camera to stop collecting data. Once the security camera receives the message, it returns back to the standby state.

In this process, since all the devices in the house are connected with the same home network, the motion sensor (with an address of 15 in decimal number) can send notifications to the security camera (with an address of 16 in decimal number) with its intra-domain address directly. Because the number of devices in the home network is small, 8-bit short addresses could be utilized to reduce the energy consumption. In addition, in this scenario, the motion sensor only needs to advertise to the security camera in a unidirectional way. Therefore, the source address could be omitted to further reduce the packet overhead and improve information transmission efficiency.

When the security camera intends to send the collected image to the host's smartphone, the packets of the image

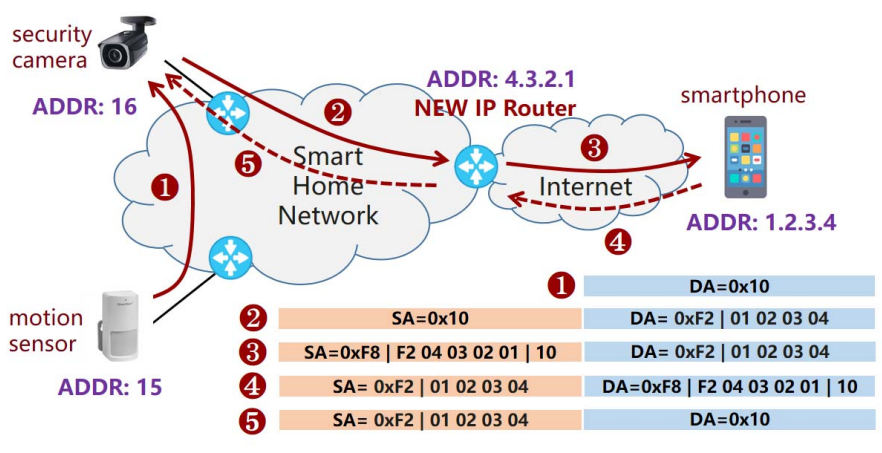

Fig. 2. An Example for Home Automation.

should be transmitted to the residential border router firstly. The reason is that the source address of this packet is not reachable in the destination network. Therefore, the NEW IP router will add an address segment (4.3.2.1 in dotted decimal notation) to the source address for the reverse routing. Then, modified data packets are sent to the user's smartphone via the Internet.

After the host understands the situation, he will send a command back to the camera to stop image collection. Once this command arrives at the residential border router, the first address segment (4.3.2.1 in dotted decimal notation) of the destination address will be removed by the NEW IP router, and the command will be sent to the security camera by the second short address segment (16 in decimal number).

In this scenario, NEW IP shows two advantages compared to the conventional IP framework. On one hand, short address space is adopted for IoT communications in the smart home network, which reduces packet overhead, thus improves the communication efficiency. On the other hand, the address segmentation enabled by NEW IP's flexibility would greatly simplify the design of the border router, because the border router does not need to maintain the address mapping instructions.

Comparing with 6LoWPAN, the proposed NEW IP framework make routers forward data packets directly based on short addresses, without any compression or decompression processes that are adopted by 6LoWPAN. In this way, NEW IP can simplify the implementation of network nodes, which is especially valuable for resource-constrained IoT networks.

\section{B. Service-aware Routing}

In this subsection, the service-aware routing scenario is illustrated to show the advantages of multi-semantic addressing and user-definable networking.

A service address uniquely identifies a service (e.g., a content or a computation instance) in the network. A server that hosts one or more services advertises the corresponding service addresses to the network. When a client tries to obtain a service, it can directly encapsulate the corresponding service address into the NEW IP header as destination address, which skips the DNS query and reduces the latency it brings. More- 
over, the metadata field could carry extra service information to meet user's requirements.

The service address format is shown in Fig. 3, where the first octet of the address is $0 \mathrm{xF} 6$, and the following octet is the type code indicating the type of the address, i.e., 0x02 for service address. The third octet represents the length of the service address. For instance, $0 x F 3$, means that an 8-byte address is followed. Therefore, the rest 8 bytes of the address is the service address itself, representing the Service 1 in Fig. 3. Besides, the high bandwidth requirement is indicated by the metadata field of the packet.

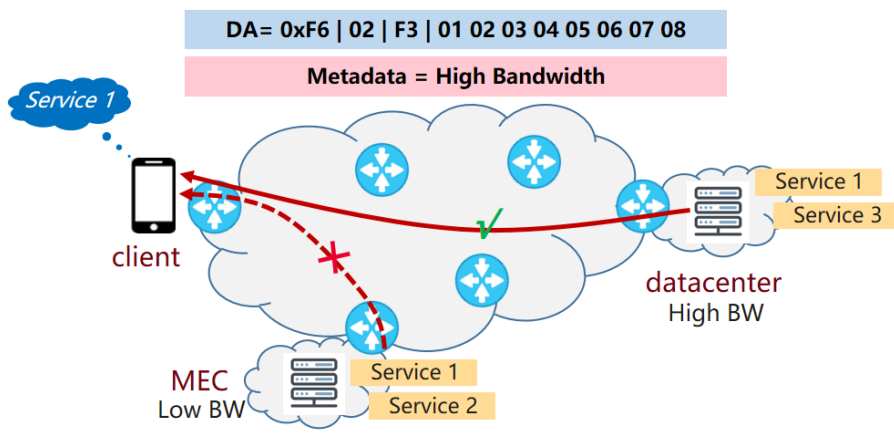

Fig. 3. Routing Based on Needs of Services.

To enable service-aware routing, the basic information of a specific service should also be advertised to the network along with its service address. Such information includes but not limited to the condition of the server, (eg. the load of the server and the connection numbers for the service), and the transmission condition of servers (e.g., bandwidth). Those information will be considered when the network calculates the corresponding routing table for the service addresses.

Fig. 3 further illustrates the details of service-aware routing, where the server both in MEC and datacenter can provide Service 1. Although the server in MEC provides the Service 1 with shorter latency, the server cannot satisfy with high bandwidth service requirement. On the other hand, the server in datacenter can provide the same service with high bandwidth and acceptable latency. Thus, the server in MEC should be chosen among all the available servers which can provide the Service 1. The NEW IP routers in the network should make the best routing choice, which is derived from a combination of multiple metrics such as server bandwidth and path latency.

In this use case, NEW IP shows the following benefits compared with today's IPv4 and IPv6 protocols:

- Faster service delivery. NEW IP enables the client to communicate with its server directly based on the service address, without querying the DNS. As introduced in [11], DNS query delay consumes up to $13 \%$ of page load time. Therefore, service-aware routing achieves a much faster service delivery by eliminating the DNS query delay;

- Support dynamic deployment of services/contents. NEW IP works in a service-centric way, which cares about "what" but not "where". This is different from the exist- ing IP routing, which must know the location (identified by the IP address) of the service previously.

- Service/application aware. NEW IP enables the network to be aware of the service-related information, which is used to calculate routing tables. In this way, service provision no longer works in a best-effort way. More information about service/application can be considered with network conditions together to achieve a better QoE for users and/or guarantees for KPIs or QoS as required for future precision services.

- Flexibility in programming of IP's header in order to provide on-demand different capabilities for network nodes on how to process packets [19].

Note that ICN networks such as NDN and MobilityFirst also work in the service-centric (or content-centric) way. However, as mentioned before, NDN's hierarchical naming schema would increase the complexity of performing lookups in routers, and MobilityFirst's fixed-length address is not suitable for all network environments, especially for resourceconstrained IoT or satellite networks.

\section{COnClusion And Future Work}

In this paper, we proposed the NEW IP, a novel IP framework for future applications. NEW IP inherently enables variable-length and multi-semantic addresses at the network layer, and user-definable networking. As such NEW IP would support better and efficiently emerging network precision applications, that provide for KPIs guarantees such as Many Networks and Holographic Communication. We presented corresponding use cases in the paper to illustrate how NEW IP achieves flexible addressing, service-aware routing and enabling customized functions to be performed on data packets. Although the flexibility in programming of NEW IP's header may possibly raise new challenges and risks to reduce the performance of network devices, we believe that modern network programming technologies such as P4 [12] and POF [13] could potentially mitigate those risks. Note that this is an initial work on this research direction, towards which we are trying to stimulate more researches as well as discussions. In the next steps, we are going to realize a design of the NEW IP framework and protocol, validate it in real network environments, and elaborate on NEW IP in-network manageability. In addition, a definition of a migration layer that will allow legacy IP-based applications and IP-Services to be used with NEW IP will be devised.

\section{REFERENCES}

[1] M. Ammar, "Ex Uno Pluria: The Service-Infrastructure Cycle Ossification and the Fragmentation of the Internet", ACM SIGCOMM Computer Communication Review, vol. 48, no. 1, pp. 56-63, 2018.

[2] Y. Liu, H. Dong, L. Zhang, and A. El Saddik, "Technical Evaluation of HoloLens for Multimedia: A First Look", IEEE MultiMedia, vol. 25, no. 4, pp. 8-18, 2018.

[3] C. Gomez, J. Paradells, C. Bormann, and J. Crowcroft, "From 6LoWPAN to 6Lo: Expanding the Universe of IPv6-Supported Technologies for the Internet of Things", IEEE Communications Magazine, vol. 55, no. 12 , pp. $148-155,2017$. 
[4] R. Li, A. Clemm, U. Chunduri, L. Dong, and K. Makhijani, "A New Framework and Protocol for Future Networking Applications", ACM SIGCOMM 2018 Workshop on Networking for Emerging Applications and Technologies (NEAT 2018), August 2018.

[5] B. Ahlgren, C. Dannewitz, C. Imbrenda, D. Kutscher, and B. Ohlman, "A Survey of Information-Centric Networking", IEEE Communications Magazine, vol. 50, no. 7, pp. 26-36, 2012.

[6] S. Ren, D. Yu, Y. Tian, X. Gong, and R. Moskowitz, "Routing and Addressing with Length Variable IP Address", ACM SIGCOMM 2019 Workshop on Networking for Emerging Applications and Technologies (NEAT 2019), pp. 21-26, 2018

[7] A. Afanasyev, J. Burke, T. Refaei, L. Wang, B. Zhang, and L. Zhang, "A Brief Introduction to Named Data Networking", 2018 IEEE Military Communications Conference (MILCOM), pp. 605-611, 2018.

[8] S. S. Adhatarao, J. Chen, M. Aurmaithurai, X. Fu, and K. K. Ramakrishnan, "Comparison of Naming Schema in ICN", 2016 IEEE International Symposium on Local and Metropolitan Area Networks (LANMAN), June 2016.

[9] D. Raychaudhuri, K. Nagaraja, and A. Venkataramani, "MobilityFirst: A Robust and Trustworthy Mobility-Centric Architecture for the Future Internet", ACM SIGMOBILE Mobile Computing and Communications Review, vol. 16, no. 3, pp. 2-13, 2012.

[10] C. Bormann, and P. Hoffman, "Concise Binary Object Representation (CBOR)", IETF RFC, vol. 7049, October 2013.

[11] X. Li, B. Liu, Y. Chen, Y. Xiao, J. Tang, and X. Wang, "Artemis: A Practical Low-Latency Naming and Routing System", in 48th International Conference on Parallel Processing (ICPP 2019), August 2019.

[12] P. Bosshart, D. Daly, G. Gibb, M. Martin and et al., "P4: Programming Protocol-Independent Packet Processors", ACM SIGCOMM Computer Communication Review, July 2014.

[13] H. Song, "Protocol-oblivious Forwarding: Unleash the Power of SDN through a Future-Proof Forwarding Plane", ACM SIGCOMM 2013 Workshop on Hot Topics in Software Defined Networking (HotSDN '13), August 2013.

[14] C. Hickman, and F. Wang, "A Variable Length Address Assignment Scheme for 6LoWPAN", 2019 IEEE 20th International Symposium on A World of Wireless, Mobile and Multimedia Networks (WoWMoM), August 2019.

[15] M. R. Sabir, M. S. Mian, K. Sattar, and M. A. Fahiem, "IP Address Space Management using Aggregated Fixed Length Subnet Masking", 2007 International Conference on Electrical Engineering, August 2007.

[16] K. Ryu, and B. Choe, "A novel address pointer switch architecture for variable length packets", 2008 10th International Conference on Advanced Communication Technologym, April 2008.

[17] G. Bouabene, C. Jelger, C. Tschudin, S. Schmid, A. Keller, and M. May, "The autonomic network architecture (ANA)", IEEE Journal on Selected Areas in Communications, vol. 28, no. 1, pp. 4-14, 2010.

[18] Y. Kang, B. Jung, "IPv6 Anycast Routing aware of a Service Flow", 2007 IEEE International Symposium on Consumer Electronics, June 2007.

[19] A. Galis, S. Denazis, C. Brou, and C. Klein, "Programmable Networks for IP Service Deployment", Artech House, 2004. 